\title{
Improved outcome by an adjunctive treatment with an IgM enriched immunoglobulin in juvenile sepsis - a single center case series
}

\section{INTRODUCTION}

Sepsis is a major cause of morbidity and mortality in critically ill patients ${ }^{1,2}$ and remains a life threatening event in adult and pediatric patients despite improvements in sepsis management in the recent years. Immunoglobulins play an important role in rebalancing the host response to an infection ${ }^{3}$.

Next to their anti-inflammatory and antibacterial activities, immunomodulation and toxin scavenging, it controls to some extent the excessive host response to an infection during sepsis. Compared to standard intravenous immunoglobulins (IVIGs), immunoglobulins with an IgIM enriched content (IVIGM) had shown a more potent immunomodulation ${ }^{4,5}$. In the current study such an IVIGM preparation (Pentaglobin $\circledast$ ) was investigated as an adjunctive treatment for juvenile patients with severe bacterial infections

\section{$\mathrm{AIM}$}

A randomized controlled clinical trial previously conducted in our center yielded encouraging results in the treatment of pediatric sepsis with IVIGM when administered alongside with standard therapy.

The aim of this data analysis was to assess the efficacy of an IgM-enriched immunoglobulin preparation as adjuvant therapy in the treatment of pediatric patients with sepsis in comparison to patients tre ated with standard therapy only. A secondary goal was to asses whether the positive treatment results seen in the previous trial could be confirmed and hence the combined results could serve as a basis for planning a larger clinical trial in the future.
This preparation is the only approved IVIGM for treating severe bacterial infections and contains the immunoglobulins IgM (12\%), IgG (76\%), and IgA (12\%). In 2014 a trial was performed to assess the efficacy of IVIGM as adjuvant therapy in the treatment of pediatric patients with sepsis in Albania. Subsequent after completion of the trial, data from patients being treated with or without IVIGM were documented.

It has been assumed that the administration of IVIGM in combination with standard-of-care antibiotic would increase the overall survival rate in juvenile septic patients admitted to the pediatric intensive care unit (PICU) as opposed to standard of care treatment only.

\section{RESULTS}

Both groups were comparable at baseline. Similar values were documented with the exception of blood pressure which was higher in the IVIGM group compared to the antibiotic (AB) group. There were no statistically significant differences with regards to age, body weight, gender distribution disease te blood cell count (WBC), platelets, and base excess (BE). The PICU survival rate was higher in the IVIGM group $(90 \%, N=40)$ than in the control group $(45 \%, N=20, P<0.0001$ in the Chi-square test).

The mean time to mortality in the control group was 18,05 days and 20,65 days in the AB plus IVIGM group. They showed a significant difference in the log rank test $P<0.015$. Differences in CRP levels between day one in the AB group. Platelet count increased from day one until day of discharge in the IVIGM group but not in the AB group. Platelet count on day of discharge was significantly different betwe
counts did not differ between groups when comparing day one and day of discharge.

In regards of safety, no adverse drug reactions concerning the IVIGM treatment were reported.

\section{METHODS}

In this data analysis, 60 consecutive juvenile septic patients admitted to the pediatric intensive care unit (PICU) at the University Hospital Center Mother Teresa in Tirana, Albania, were included. Inclusion period was between Ist Jan 2014 to 30 h Dec 2016. All patients were treated according to standard PICU sepsis guidelines with antibiolics. Upon availablity in our ward, IVGM (Penlaglobin $\circledast$, Biotest AG, Germany) was given as an adjuvant treatment to standard therapy (40 patients). 20 patients have been treated with standard therapy only (AB group).

The study was approved by the local Ethics committee in Tirana as part of Sepsis guideline treatment.

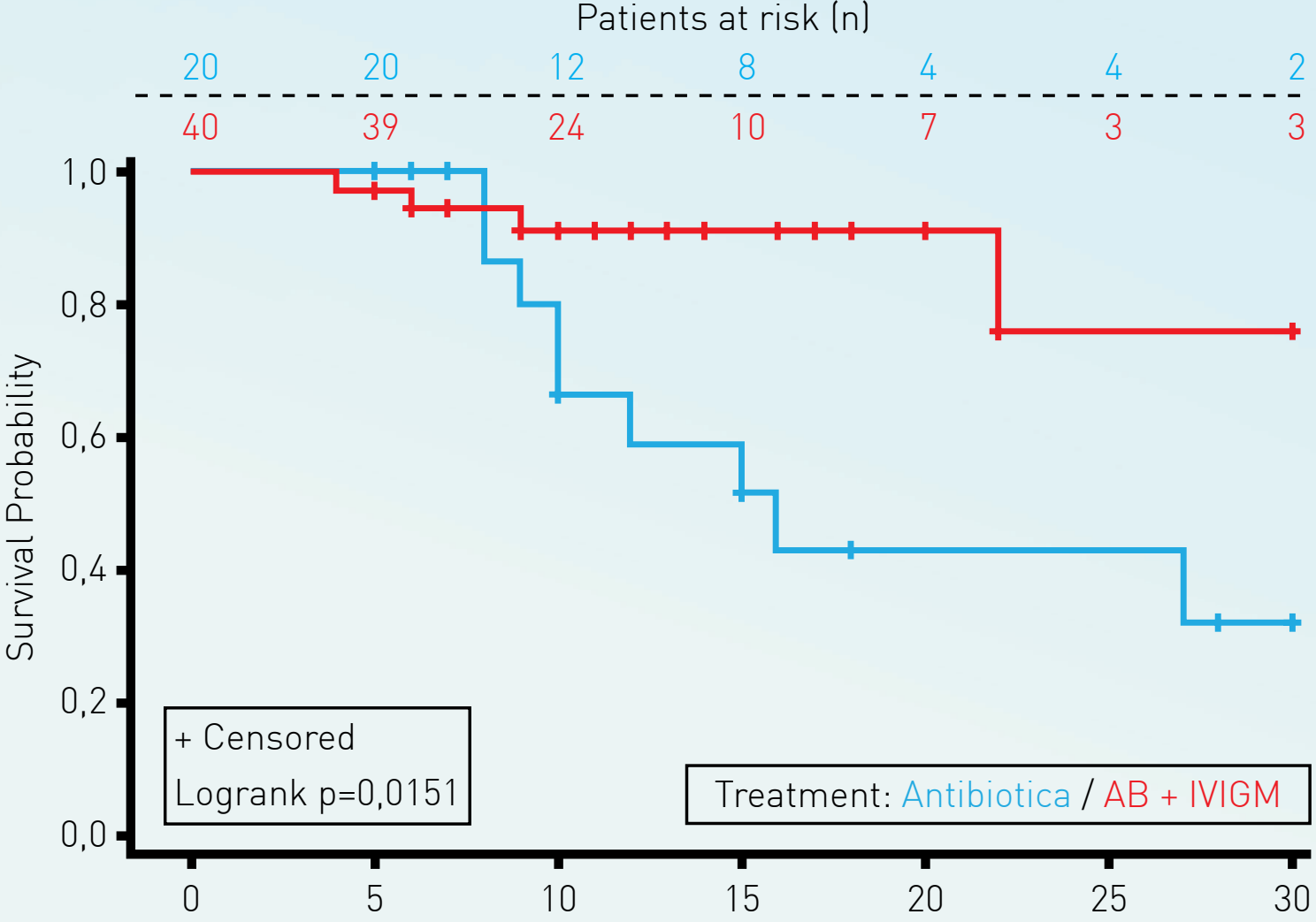

Fig. 1 Kaplan Meyer Survival analysis of the antibiotic

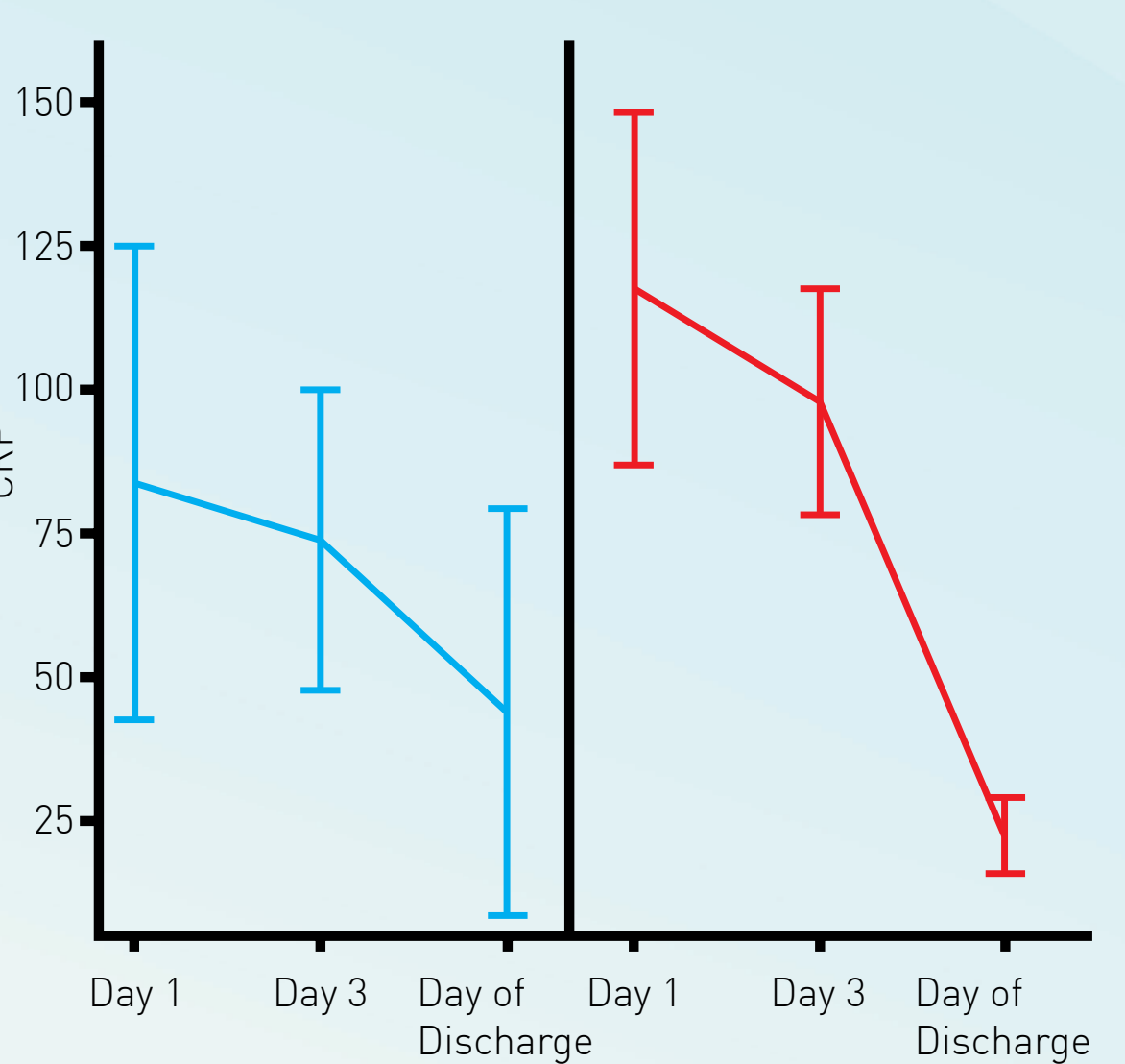

Fig. 2 Change in CRP over time

\begin{tabular}{|c|c|c|c|c|c|c|c|c|c|}
\hline Baseline & Age in Years & Weight in $\mathrm{kg}$ & CRP in $\mathrm{mg} / \mathrm{ml}$ & WBC in $\mathrm{mm}^{3}$ & Platelets $\times 10^{9} / \mathrm{L}$ & $\mathrm{BE}$ in $\mathrm{mmol} / \mathrm{L}$ & $\mathrm{BP}$ in $\mathrm{mmHg}$ & $\begin{array}{c}\text { Gender } \\
\text { Distribution }\end{array}$ & Distribution AVPU \\
\hline & Mean & Mean & Mean & Mean & Mean & Mean & Mean Systolic & $\mathrm{M} / \mathrm{F}$ in \% & $\mathrm{AV} / \mathrm{PU}$ in \% \\
\hline AB Group & 2.21 & 11.56 & 83.48 & 16.6 & 224.1 & -5.5 & 87.55 & $35 / 65$ & $65 / 35$ \\
\hline$A B+I V I G M$ & 3.1 & 13.43 & 118 & 15.03 & 301.3 & -4.99 & 96.83 & $47.5 / 52.5$ & $82.5 / 17.5$ \\
\hline $\mathrm{p}$ Value & $>0.05$ & $>0.05$ & $>0.05$ & $>0.05$ & $>0.05$ & $>0.05$ & 0.001 & $>0.05$ & $>0.05$ \\
\hline
\end{tabular}

Tab. 1 Demographics and baseline values

\begin{tabular}{|c|c|c|c|c|c|c|c|}
\hline Parameter & Treatment & Day 1 & Day 3 & Day of Discharge & Change Day 1 to Day 3 & $\begin{array}{c}\text { Change Day } 1 \text { to Day of } \\
\text { Discharge }\end{array}$ & $\begin{array}{c}A B \text { versus } A B+I V I G M \\
\text { at Day } 3\end{array}$ \\
\hline \multirow[t]{2}{*}{ CRP $[\mathrm{mg} / \mathrm{ml}]$} & $A B$ & 83.6 & 73.9 & 44.0 & ns & ns & ns \\
\hline & $A B+I V I G M$ & 106.5 & 98.8 & 22.9 & ns & $p<0.05$ & \\
\hline \multirow[t]{2}{*}{ Platelets $\left[\times 10^{5}\right]$} & $A B$ & 2.2 & 1.7 & 2.1 & ns & ns & $<0.005$ \\
\hline & $A B+I V I G M$ & 3.0 & 3.3 & 3.9 & ns & ns & \\
\hline \multirow[t]{2}{*}{ WBC $\left[\times 10^{3}\right]$} & $A B$ & 16.6 & 12.6 & 11.3 & ns & ns & ns \\
\hline & $A B+I V I G M$ & 15.5 & 14.6 & 11.6 & ns & ns & \\
\hline
\end{tabular}

\section{DISCUSSION}

The data shown here have several limitations. No standardized antibiotic treatment was given to all patients. This is a consecutive case collection where the treatment allocation to the $A B$ and the $A B+$ IVIGM treatment was driven by the availability of IVIGM and not by the study protocol defined treatment allocation such as randomization. Despite this uncommon treatment allocation, baseline data in both treatment arms are comparable. Only for blood pressure (BP) a difference was
gest difference was seen in the subgroup of patients below one year of age.

This analysis documents the use of IVIGM under daily clinical routine conditions and can help close knowledge gaps with regards to juveniles with sepsis. Published data in juvenile sepsis with adjunctive differents buch as IVIGM are scarce. The results found $55 \%$ m this case series show an even larger IVIGM with $10 \%$ mortality in regards to the PICU all cause mortality, than it was reported for the RCT conducted in our institution $(35.9 \% \text { versus } 12.8 \%)^{\circ}$.

The mortality rate in the IVIGM group was low and similar in both studies with $10 \%$ and $12.8 \%$. Overall the data seem to confirm the good efficacy of IgM-enriched immunoglobulin in a juvenile patient population suffering from sepsis.

\section{CONCLUSION}

Tolerability of the IVIGM was very good, no adverse drug reactions caused by the IVIGM treatment were reported.

The results of this retrospective data analysis represent real life clinical experience and confirm the benefit in the reduction of the mortality rate in juvenile sepsis by the adjunctive treatment with IVIGM we have found in our randomized controlled clinical trial previously. 